\title{
Stability of non-monotone critical waves in a population dy- namics model with spatio-temporal delay
}

\author{
Yong-Hui Zhou a,*, Yun-Rui Yang ${ }^{\mathrm{b}}$, Hong-Juan Zhang ${ }^{\mathrm{c}}$ \\ a School of Mathematics and Statistics, Hexi University, Zhangye, Gansu 734000, P. R. China. \\ ${ }^{b}$ School of Mathematics and Physics, Lanzhou Jiaotong University, Lanzhou, Gansu 730070, P. R. China. \\ ${ }^{c}$ School of Agricultural and Biological Technology, Hexi University, Zhangye, Gansu 734000, P. R. China.
}

Communicated by I. Koca

\begin{abstract}
This paper, we show the stability of non-monotone critical waves by a anti-weighted method for a kind of non-monotone time-delayed reaction-diffusion equations including Nicholson's blowflies equation which describes the population dynamics of a single species with age structure.
\end{abstract}

Keywords: Delay, critical waves, anti-weighted method, stability.

2010 MSC: 34K18, 37G10, 37G05.

(C)2018 All rights reserved.

\section{Introduction and preliminaries}

In this paper, we study the stability of non-monotone critical traveling waves for the following nonlocal delayed reaction-diffusion equation

$$
\frac{\partial u}{\partial t}=D \frac{\partial^{2} u}{\partial x^{2}}-d u(t, x)+\int_{\mathbb{R}} h(y) b(u(t-\tau, x-y)) d y, t>0, x \in \mathbb{R}
$$

with the initial data

$$
u(s, x)=u_{0}(s, x), \quad(s, x) \in[-\tau, 0] \times \mathbb{R},
$$

where $\tau \geqslant 0, h(x)$ is non-negative, unit, and symmetric kernels, $b(u)$ is the birth rate function, which are assumed to satisfy the following hypothesis:

$\left(\mathrm{H}_{1}\right) \mathrm{b}(\mathrm{u})$ is a non-negative, $\mathrm{C}^{2}$ smooth increasing function, and satisfies $\left|\mathrm{b}^{\prime}(u)\right| \leqslant \mathrm{b}^{\prime}(0)$ for $u \in[0, \infty)$;

\footnotetext{
*Corresponding author

Email addresses: 2823877618@qq. com (Yong-Hui Zhou), lily1979101@163.com (Yun-Rui Yang)

doi: $10.22436 / \mathrm{mns} .02 .01 .02$
}

Received: 2017-11-21 Revised: 2017-12-19 Accepted: 2018-04-07 
$\left(\mathrm{H}_{2}\right)$ Two constant equilibria of (1.1): $\mathrm{u}_{-}=0$ is unstable and $\mathrm{u}_{+}$is stable, namely, $\mathrm{d}-\mathrm{b}^{\prime}(0)<0$ and $\mathrm{d}-\mathrm{b}^{\prime}\left(\mathrm{u}_{+}\right)>0$

$\left(\mathrm{H}_{3}\right)$ There exists $a \mathfrak{u}_{*} \in\left[0, u_{+}\right]$such that $b(\cdot)$ is increasing on $\left[0, u_{*}\right]$ and decreasing on $\left[\mathfrak{u}_{*}, \mathfrak{u}_{+}\right]$. In particular, $\mathrm{b}^{\prime}(0)>0$ and $\mathrm{b}^{\prime}\left(\mathrm{u}_{+}\right)<0$;

$\left(\mathrm{H}_{4}\right) \mathrm{h}$ is nonnegative integrable, $\int_{\mathbb{R}} h(y) d y=1$, and $h(-x)=h(x), \forall x \in \mathbb{R}$;

$\left(\mathrm{H}_{5}\right) \int_{\mathbb{R}} e^{-\lambda_{*}\left(y+c_{*} \tau\right)} h(y) d y<\infty$.

There are two well-known examples of the equation (1.1) satisfying $\left(\mathrm{H}_{1}\right)-\left(\mathrm{H}_{5}\right)$. One is the so-called nonlocal dispersion Nicholson's blowflies equation [3, 4] with

$$
b(u)=p u e^{-a u}, \text { for } a>0, p>0,
$$

where $u_{-}=0, u_{+}=\frac{1}{a} \ln \frac{p}{d}$. When $\frac{p}{d}>e$, the birth rate function $b(u)$ is a uni-modality function with the maximum at $\mathfrak{u}_{*}:=\frac{1}{a} \in\left(0, u_{+}\right)$, and it can be verified that $\left|b^{\prime}(u)\right| \leqslant b^{\prime}(0)$ for $u \in[0, \infty)$. The other is the so-called nonlocal dispersion Mackey-Glass equation [10, 11] with

$$
b(u)=\frac{p u}{1+a u^{q}}, \text { for } a>0, p>0,
$$

where $u_{-}=0, u_{+}=\left(\frac{p-d}{d a}\right)^{\frac{1}{q}}$. When $\frac{p}{d}>\frac{q}{q-1}$, the birth rate function $b(u)$ is non-monotone with unimodality at $u_{*}=:[a(q-1)]^{-\frac{1}{q}} \in\left(0, u_{+}\right)$, and it can be verified that $\left|b^{\prime}(u)\right| \leqslant b^{\prime}(0)$ for $u \in[0, \infty)$.

The traveling waves for (1.1) connecting two steady states $u_{ \pm}$at far fields are the special solutions to (1.1) in the form of $u(t, x)=\phi(x+c t)$, namely,

$$
\left\{\begin{array}{l}
c \phi^{\prime}(\xi)=D \phi^{\prime \prime}(\xi)-d \phi(\xi)+\int_{\mathbb{R}} h(y) b(\phi(\xi-y-c \tau)) d y \\
\phi(-\infty)=0, \quad \phi(+\infty)=\mathrm{K} .
\end{array}\right.
$$

The existence, uniqueness and asymptotic behavior of the non-monotone traveling waves of (1.1) have been guaranteed by [18]. Our study includes the Mei's [1] work. We briefly describe the results we need below.

(i) Behavior of $\phi(\xi)$ for $\xi \sim-\infty$. Since $\phi(\xi) \rightarrow u_{-}$as $\xi \rightarrow-\infty$, we expect that $\phi(\xi)$ is close to a function $u(\xi)$ which satisfies the linearized equation of (1.5) around $u_{-}$for $\xi \sim-\infty$ :

$$
c u^{\prime}(\xi)-D u^{\prime \prime}(\xi)+d u(\xi)=b^{\prime}(0) \int_{\mathbb{R}} h(y) \phi(\xi-y-c \tau) d y, \quad u(-\infty)=0 .
$$

By substituting $u(\xi)=e^{\lambda \xi}$ to the above linearized equation, we obtain the following characteristic equation for a pair of $(c, \lambda)$ :

$$
c \lambda-D \lambda^{2}+d=b^{\prime}(0) \int_{\mathbb{R}} h(y) e^{-\lambda(y+c \tau)} d y .
$$

To investigate the admission of $(c, \lambda)$ to the above characteristic equation, we denote

$$
G_{c}(\lambda):=c \lambda-D \lambda^{2}+d, \quad H_{c}(\lambda):=b^{\prime}(0) \int_{\mathbb{R}} h(y) e^{-\lambda(y+c \tau)} d y .
$$

As shown in [10] that, for each $\tau>0$, there exists a unique $c_{*}=c_{*}(\tau)>0$ at which the two graphs of $G_{c}$ and $\mathrm{H}_{\mathrm{c}}$ are tangent at $\lambda_{*}$. This means that $\left(\mathrm{c}_{*}, \lambda_{*}\right)$ are uniquely determined by

$$
\mathrm{G}_{\mathfrak{c}_{*}}\left(\lambda_{*}\right)=\mathrm{H}_{\mathfrak{c}_{*}}\left(\lambda_{*}\right), \quad \mathrm{G}_{\mathfrak{c}_{*}}^{\prime}\left(\lambda_{*}\right)=\mathrm{H}_{\mathfrak{c}_{*}}^{\prime}\left(\lambda_{*}\right) .
$$

Thus, we obtain

(1) for $c>c_{*}$, the characteristic equation (1.6) has two distinct solutions $0<\lambda_{1}<\lambda_{2}$; 
(2) for $\mathrm{c}=\mathrm{c}_{*}$, (1.6) has multiple roots $\lambda_{1}=\lambda_{2}=\lambda_{*}$;

(3) for $c<c_{*}$, (1.6) has no positive root.

When $c<c_{*}$, there will be no traveling wave for Eq. (1.5). For it would satisfy the linearized equation for $\xi \sim-\infty$, and would have the form $e^{\lambda \xi}$ for $\xi \sim-\infty$, but no such can exist. When $c \geqslant c_{*}>0$, on the other hand, the traveling wave $\phi(x+c t)$, if exists, should satisfy

$$
\left\{\begin{array}{l}
\phi(\xi)=\mathrm{O}(1) e^{\lambda_{1} \xi} \rightarrow 0 \text { as } \xi \rightarrow-\infty, \text { for } c>c_{*}, \\
\phi(\xi)=\mathrm{O}(1)|\xi| e^{\lambda_{*} \xi} \rightarrow 0 \text { as } \xi \rightarrow-\infty, \text { for } c=c_{*} .
\end{array}\right.
$$

(ii) Behavior of $\phi(\xi)$ for $\xi \sim-\infty$. The asymptotic behavior of the traveling wave $\phi(\xi)$ at $\xi=\infty$ is solely determined by the linearized ODE around $u_{+}$:

$$
c\left(\phi-u_{+}\right)^{\prime}-D\left(\phi-u_{+}\right)^{\prime \prime}+d\left(\phi-u_{+}\right)=b^{\prime}\left(u_{+}\right) \int_{\mathbb{R}} h(y)\left(\phi(\xi-y-c \tau)-u_{+}\right) d y .
$$

Let $\phi-u_{+}=e^{-\lambda_{+} \xi}$ as $\xi \rightarrow+\infty$, we get

$$
-c \lambda_{+}-D \lambda_{+}^{2}+d=b^{\prime}\left(u_{+}\right) \int_{\mathbb{R}} h(y) e^{\lambda_{+}(y+c \tau)} d y,
$$

which uniquely solves for $\lambda_{+}=\lambda_{+}(\mathrm{c})>0$ and $\lambda_{+}^{*}=\lambda_{+}^{*}\left(\mathrm{c}_{*}\right)>0$. Therefore, the asymptotic behavior of the traveling wave as $\xi \rightarrow \infty$ is

$$
\left|u_{+}-\phi(\xi)\right|=\left\{\begin{array}{l}
\mathrm{O}(1) e^{-\lambda_{+} \xi} \rightarrow 0 \text { as } \xi \rightarrow \infty, \text { for } c>c_{*}, \\
\mathrm{O}(1) e^{-\lambda_{+}^{*} \xi} \rightarrow 0 \text { as } \xi \rightarrow \infty, \text { for } c=c_{*} .
\end{array}\right.
$$

(iii) Existence, uniqueness, monotone/oscillatory of the traveling waves.

(1). When $d \geqslant\left|b^{\prime}\left(u_{+}\right)\right|$, the traveling wave $\phi(x+c t)$ exists uniquely (up to a shift) for every $c \geqslant c_{*}=c_{*}(\tau)$, where the time-delay $\tau$ is allowed to be any number in $[0, \infty)$. If $0 \leqslant \tau<\tau$, then these traveling waves are monotone; while, if $\tau \geqslant \underline{\tau}$, then the traveling waves are still monotone for $(c, \tau) \in\left[c_{*}, c^{*}\right] \times\left[\underline{\tau}, \tau_{0}\right]$, where $\mathrm{c}_{*}=\mathrm{c}_{*}(\tau)$ is the minimum wave speed as mentioned before, $\mathrm{c}^{*}=\mathrm{c}^{*}(\tau)$ is given by the characteristic equation for (1.5) around $u_{+}$, and $\tau_{0}(>\tau)$ is the unique intersection point of two curves $c_{*}(\tau)$ and $c^{*}(\tau)$; and the traveling waves are oscillating around $u_{+}$for $(c, \tau)\left[c_{*}, c^{*}\right] \times\left[\tau, \tau_{0}\right]$, namely, either $c>c^{*}$ or $\tau>\tau_{0}$ [6].

(2). When $d<\left|b^{\prime}\left(u_{+}\right)\right|$, on the other hand, the traveling wave $\phi(x+c t)$ with $c \geqslant c_{*}$ can exist only when $\tau<\bar{\tau}$, and no traveling wave can exist for $\tau \geqslant \bar{\tau}$. In the case of $\tau<\bar{\tau}$, the waves are monotone for $0<\tau<\underline{\tau}$ and oscillating for $\tau \in(\underline{\tau}, \bar{\tau})[6]$, where $\bar{\tau}, \underline{\tau}$ are positive constants.

When the birth function $f$ is monotone, authors in $[1,2,6,7,10-12,14]$ investigated the existence of monotone traveling waves by using the monotone iteration and fixed-points theorem with help of the upper-lower solutions. By using the Fourier transform, Green's function and the weighted energy method, the authors in $[13,16,17]$ showed the global stability of critical traveling waves, which depends on the monotonicity of both the equation and traveling waves. For the stability of traveling wave solutions, it is always one of the important and difficult objects in the theory of traveling waves. In the case of monotone systems, the squeezing technique, the weighted energy method combining comparison principle, the convergence theory for monotone semiflows, and spectral analysis method are used usually to solve the the stability of monotone traveling wave solutions. Very recently, for the local Nicholson's blowflies equation, Mei [6] succeeded in obtaining the stability of all monotone/non-monotone traveling waves with $c>c_{*}$, by means of the regular $\mathrm{L}_{2}$-weighted energy method with a new development by a nonlinear Halanay's inequality.

However, for the non-monotone and critical case, the stability results of traveling waves are respectively limited, because comparison principle does not still hold for the scalar equation or system without 
monotonicity. Inspired by the study on classical Fisher-KPP equation by Moet [15], Chern et al. [1] where they introduced a suitable transform function (or say, an anti-weight) to switch the equation to a new equation (we call it the anti-weighted energy method), we study the stability of the non-monotone critical traveling waves for (1.1).

Notations: Throughout this paper, $C>0$ denotes a generic constant, $C_{i}>0(i=1,2, \ldots)$ represents a specific constant. Let I be an interval. $\mathrm{L}^{2}(\mathrm{I})$ is the space of the square integrable functions defined on $\mathrm{I}$, and $H^{k}(I)(k \geqslant 0)$ is the Sobolev space of the $L^{2}$-functions $f(x)$ defined on the interval I whose derivatives $\frac{d^{i}}{d x^{i}} f(i=1,2, \ldots, k)$ also belong to $L^{2}(I) . L_{w}^{2}(I)$ denotes the weighted $L^{2}$-space with a weight function $\mathcal{w}(x)>0$ and its norm is defined by

$$
\|f\|_{L_{w}^{2}}=\left(\int_{I} w(x)|f(x)|^{2} d x\right)^{\frac{1}{2}}
$$

$\mathrm{H}_{w}^{k}(\mathrm{I})$ is the weighted Sobolev space with the norm given by

$$
\|f\|_{H_{w}^{k}}=\left(\sum_{i=0}^{k} \int_{I} w(x)\left|\frac{d^{i}}{d x^{i}} f(x)\right|^{2} d x\right)^{\frac{1}{2}} .
$$

Let $T>0$ be a number and $\mathcal{B}$ be a Banach space. We denote by $C([0, T] ; \mathcal{B})$ the space of the $\mathcal{B}$-valued continuous functions on $[0, T] . L^{2}([0, T] ; \mathcal{B})$ as the space of the $\mathcal{B}$-valued $\mathrm{L}^{2}$-functions on $[0, T]$. The corresponding spaces of $\mathcal{B}$-valued functions on $[0, \infty)$ are defined similarly.

The rest of this paper is organized as follows. In Section 2, we introduce some preliminaries and state our stability result. In Section 3, we prove the global existence and uniqueness of the solution for the perturbed equation, where the initial perturbation can be allowed to be arbitrarily large. In Section 4 , when the initial perturbation is suitably small, the solution of the perturbed equation can be proved to be uniformly bounded by the anti-weighted energy method. Based on the uniform boundedness, we shall further prove the asymptotic stability in Section 5.

\section{Preliminaries and main result}

Let $\phi(\xi):=\phi\left(x+c_{*} t\right), \xi:=x+c_{*} t$ be a given critical traveling wave, and define

$$
v(t, \xi)=u(t, x)-\phi(\xi), \quad v_{0}(s, \xi)=u_{0}(s, x)-\phi\left(x+c_{*} s\right) .
$$

Then, from (1.1)-(1.5), $v(t, \xi)$ satisfies

$$
\begin{aligned}
v_{t}(t, \xi)+c_{*} \nu_{\xi}(t, \xi)-D v_{\xi \xi}(t, \xi)+d v(t, \xi) & =\int_{\mathbb{R}} h(y) P\left(v\left(t-\tau, \xi-y-c_{*} \tau\right)\right) d y, \\
v(s, \xi) & =v_{0}(s, \xi), \quad(s, \xi) \in[-\tau, 0] \times \mathbb{R},
\end{aligned}
$$

where

$$
\mathrm{P}(v)=\mathrm{b}(\phi+v)-\mathrm{b}(\phi),
$$

with $v=v\left(t-\tau, \xi-y-c_{*} \tau\right)$ and $\phi=\phi\left(\xi-y-c_{*} \tau\right)$. Furthermore, let us linearize the (2.1), we equivalently obtain

$$
\begin{aligned}
v_{t}(t, \xi) & +c_{*} v_{\xi}(t, \xi)-D v_{\xi \xi}(t, \xi)+d v(t, \xi)-\int_{\mathbb{R}} h(y) b^{\prime}\left(\phi\left(\xi-y-c_{*} \tau\right)\right) v\left(t-\tau, \xi-y-c_{*} \tau\right) d y \\
& =\int_{\mathbb{R}} h(y) Q\left(v\left(t-\tau, \xi-y-c_{*} \tau\right)\right) d y
\end{aligned}
$$


where

$$
\mathrm{Q}(v)=\mathrm{b}(\phi+v)-\mathrm{b}(\phi)-\mathrm{b}^{\prime}(\phi) v
$$

with $v=v\left(t-\tau, \xi-y-c_{*} \tau\right)$ and $\phi=\phi\left(\xi-y-c_{*} \tau\right)$, and satisfies, by Taylor's formula

$$
|\mathrm{Q}(v)|=\mathrm{O}(1)|v|^{2} \text {. }
$$

Define a weight function as

$$
\mathcal{w}(x)=e^{-2 \lambda_{*} \xi},
$$

note that $\lim _{\xi \rightarrow \infty} w(\xi)=0$ and $\lim _{\xi \rightarrow-\infty} \mathcal{w}(\xi)=+\infty$.

To deal with the delay equation, we define some spaces, for $0<\mathrm{T} \leqslant \infty$,

$$
\begin{aligned}
& C_{\text {unif }}[-\tau, T]:=\left\{u(t, x) \in C([-\tau, T] \times \mathbb{R}) \text { such that } \lim _{x \rightarrow+\infty} u(t, x) \text { exists uniformly in } t \in[-\tau, T]\right. \text { and } \\
&\left.\lim _{x \rightarrow+\infty} u_{x}(t, x)=\lim _{x \rightarrow+\infty} u_{x x}(t, x)=0, \text { uniformly with respect to } t \in[-\tau, T]\right\}, \\
& X_{0}[-\tau, 0]:=\left\{v \mid v \in C([-\tau, 0] ; C(\mathbb{R})) \cap C_{u n i f}[-\tau, 0], \sqrt{w} v \in C\left([-\tau, 0] ; H^{1}(\mathbb{R})\right)\right. \\
&\text { and } \left.\sqrt{w} v \in \mathrm{L}^{2}\left([-\tau, 0] ; \mathrm{H}^{2}(\mathbb{R})\right)\right\},
\end{aligned}
$$

with

$$
\mathrm{M}_{0}^{2}:=\sup _{\mathrm{t} \in[-\tau, 0]}\left(\|v(\mathrm{t})\|_{\mathrm{C}(\mathbb{R})}^{2}+\|(\sqrt{w} \mathcal{v})(\mathrm{t})\|_{\mathrm{H}^{1}(\mathbb{R})}^{2}\right)+\int_{-\tau}^{0}\|(\sqrt{w} v)(s)\|_{\mathrm{H}^{2}(\mathbb{R})}^{2} \mathrm{ds},
$$

and

$$
\begin{aligned}
\mathrm{X}_{\mathrm{loc}}(0, \infty):= & \left\{v \mid v \in \mathrm{C}_{\mathrm{loc}}([0, \infty) ; \mathrm{C}(\mathbb{R})) \cap \mathrm{C}_{\text {unif }}[0, \infty), \sqrt{w} v \in \mathrm{C}_{\mathrm{loc}}\left([0, \infty) ; \mathrm{H}^{1}(\mathbb{R})\right)\right. \\
& \text { and } \left.\sqrt{w} v \in \mathrm{L}_{\mathrm{loc}}^{2}\left([0, \infty) ; \mathrm{H}^{2}(\mathbb{R})\right)\right\},
\end{aligned}
$$

where $\mathrm{L}_{\text {loc }}^{2}\left([0, \infty) ; \mathrm{H}^{2}(\mathbb{R})\right)$ is the space whose $\mathrm{H}^{2}$-valued functions are locally $\mathrm{L}^{2}$-integrable in $[0, \infty)$. The locally continuous spaces $\mathrm{C}_{\mathrm{loc}}([0, \infty) ; \mathrm{C}(\mathbb{R}))$ and $\mathrm{C}_{\mathrm{loc}}\left([0, \infty) ; \mathrm{H}^{1}(\mathbb{R})\right)$ are similarly defined. We further define

$$
\begin{aligned}
\mathrm{X}(0, \infty):= & \left\{v \mid v \in \mathrm{C}([0, \infty) ; \mathrm{C}(\mathbb{R})) \cap \mathrm{C}_{\text {unif }}[0, \infty), \sqrt{w} v \in \mathrm{C}\left([0, \infty) ; \mathrm{H}^{1}(\mathbb{R})\right)\right. \\
& \text { and } \left.\sqrt{\phi w} v \in \mathrm{L}^{2}\left([0, \infty) ; \mathrm{L}^{2}(\mathbb{R})\right), \partial_{\xi}(\sqrt{w} v) \in \mathrm{L}^{2}\left([0, \infty) ; \mathrm{H}^{1}(\mathbb{R})\right)\right\}
\end{aligned}
$$

with

$$
M_{\infty}^{2}:=\sup _{t \in[0, \infty)}\left(\|v(t)\|_{C(\mathbb{R})}^{2}+\|(\sqrt{w} v)(t)\|_{H^{1}(\mathbb{R})}^{2}\right)+\int_{0}^{\infty}\|(\sqrt{\phi w} v)(s)\|_{L^{2}(\mathbb{R})}^{2} d s+\int_{0}^{\infty}\left\|\partial_{\xi}(\sqrt{w} v)(s)\right\|_{H^{1}(\mathbb{R})}^{2} d s .
$$

Now we state main results which are the global existence, uniqueness, uniform boundedness, and stability for the solution to (1.1) with a general non-monotone birth rate $b(u)$ as the following three theorems, respectively.

Theorem 2.1 (Global existence and uniqueness). Assume that $\left(\mathrm{H}_{1}\right)-\left(\mathrm{H}_{5}\right)$ hold. Let $\mathrm{b}^{\prime}\left(\mathrm{u}_{+}\right)$and $\tau$ satisfy, either $\mathrm{d} \geqslant\left|\mathrm{b}^{\prime}\left(\mathrm{u}_{+}\right)\right|$with arbitrarily given $\tau>0$, or $\mathrm{d}<\left|\mathrm{b}^{\prime}\left(\mathrm{u}_{+}\right)\right|$with $0<\tau<\bar{\tau}$. Let $\phi\left(x+\mathrm{c}_{*} \mathrm{t}\right)=\phi(\xi)$ be any given critical traveling wave, and the initial perturbation $\nu_{0}(s, \xi):=\mathfrak{u}_{0}(s, x)-\phi(\xi) \in X_{0}(-\tau, 0)$ be arbitrary, then the solution $v(t, \xi)$ of the perturbed equation (2.1) globally and uniquely exists in $\mathrm{X}_{\mathrm{loc}}(0, \infty)$.

Theorem 2.2 (Uniform boundedness). Under the conditions of Theorem 2.1, if the initial perturbation $v_{0} \in$ $\mathrm{X}_{0}(-\tau, 0)$ is small enough, namely, there exists a constant $\delta_{0}>0$ such that $\mathrm{M}_{0} \leqslant \delta_{0}$, then the solution $v(\mathrm{t}, \xi)$ of the perturbed equation (2.2) satisfies $v \in X(0, \infty)$, and $v(t, \xi)$ is uniformly bounded in $X(0, \infty)$ :

$$
\mathrm{M}_{\infty}^{2} \leqslant \mathrm{CM}_{0}^{2} .
$$

Theorem 2.3 (Stability). Under the conditions in Theorem 2.2, then it holds that

$$
\lim _{t \rightarrow \infty} \sup _{\xi \rightarrow \mathbb{R}}|v(t, \xi)|=0 .
$$




\section{Proof of global existence and uniqueness}

When $\mathrm{t} \in[0, \tau],(2.1)$ is linear, because $\mathrm{t}-\tau \in[-\tau, 0]$ such that $\mathrm{P}\left(v\left(\mathrm{t}-\tau, \xi-y-\mathrm{c}_{*} \tau\right)\right)=\mathrm{P}\left(v_{0}(\mathrm{t}-\tau, \xi-\right.$ $\left.y-c_{*} \tau\right)$ ). Thus, the solution of (2.1) can be explicitly and uniquely solved by, for $t \in[0, \tau]$

$$
\begin{aligned}
v(t, \xi)= & e^{-d t} \int_{\mathbb{R}} G(\eta, t) v_{0}(0, \xi-\eta) d \eta+\int_{0}^{t} e^{-d(t-s)} \int_{\mathbb{R}} G(\eta, t-s) \\
& \cdot \int_{\mathbb{R}} h(y) P\left(v_{0}\left(s-\tau, \xi-\eta-y-c_{*} \tau\right)\right) d y d \eta d s,
\end{aligned}
$$

where $G(\eta, t)=\frac{1}{\sqrt{4 \pi D t}} e^{-\frac{\left(\eta+c_{*} t\right)^{2}}{4 D t}}$.

When $v_{0} \in X_{0}(-\tau, 0)$, we are going to prove $v \in X_{\text {loc }}(0, \tau)$. Multiplying (2.1) by $w(\xi) v(t, \xi)$, and using Cauchy-Schwarz inequality

$$
\left|\mathrm{D} w^{\prime} v v_{\xi}\right| \leqslant \mathrm{D} w v_{\xi}^{2}+\frac{\mathrm{D}}{4}\left(\frac{w^{\prime}}{w}\right)^{2} w v^{2}
$$

we obtain

$$
\begin{aligned}
& \left\{\frac{1}{2} w v^{2}\right\}_{t}+\left\{\frac{c_{*}}{2} w v^{2}-D w v_{\xi}\right\}_{\xi}+\left\{-\frac{c_{*}}{2} \frac{w^{\prime}}{w}-\frac{D}{4}\left(\frac{w^{\prime}}{w}\right)^{2}+d\right\} w v^{2} \\
& \leqslant \int_{\mathbb{R}} h(y) P\left(v_{0}\left(t-\tau, \xi-y-c_{*} \tau\right)\right) w v d y .
\end{aligned}
$$

Integrating the above inequality with respect to $\xi$ and $t$ over $\mathbb{R} \times[0, t]$, we then get

$$
\|\sqrt{w} v\|_{\mathrm{L}^{2}}^{2}+\mathrm{m}_{1} \int_{0}^{\mathrm{t}} \int_{\mathrm{R}} w v^{2} \mathrm{~d} \xi \mathrm{d} s \leqslant 2 \int_{0}^{\mathrm{t}} \int_{\mathrm{R}} \int_{\mathbb{R}} \mathrm{h}(\mathrm{y}) \mathrm{P}\left(v_{0}\left(s-\tau, \xi-y-c_{*} \tau\right)\right) w v d y d \xi \mathrm{d} s+\left\|\sqrt{w} v_{0}(0)\right\|_{\mathrm{L}^{2}}^{2},
$$

where $m_{1}=c_{*} \lambda_{*}-D \lambda_{*}^{2}+d=b^{\prime}(0) \int_{\mathbb{R}} h(y) e^{-\lambda_{*}\left(y+c_{*} \tau\right)}>0$.

We now turn to estimate the first and second terms of right-hand-side of (3.2),

$$
\begin{aligned}
& \int_{0}^{t} \int_{\mathbb{R}} \int_{\mathbb{R}} h(y) P\left(v_{0}\left(s-\tau, \xi-y-c_{*} \tau\right)\right) w v d y d \xi d s \\
& \quad \leqslant C \int_{0}^{t} \int_{\mathbb{R}} \int_{\mathbb{R}} h(y)\left|v_{0}\left(s-\tau, \xi-y-c_{*} \tau\right)\right| w|v| d y d \xi d s \\
& \quad \leqslant \epsilon \int_{0}^{t}\|\sqrt{w} v\|_{\mathrm{L}^{2}}^{2} \mathrm{~d} s+\frac{\mathrm{C}}{4 \epsilon} \int_{0}^{\mathrm{t}} \int_{\mathbb{R}} \int_{\mathbb{R}} h(y) w\left|v_{0}\left(s-\tau, \xi-y-c_{*} \tau\right)\right|^{2} \mathrm{~d} y \mathrm{~d} \xi \mathrm{d} s \\
& \quad=\epsilon \int_{0}^{\mathrm{t}}\|\sqrt{w} v\|_{\mathrm{L}^{2}}^{2} \mathrm{~d} s+\frac{\mathrm{C}}{4 \epsilon} \int_{-\tau}^{\mathrm{t}-\tau} \int_{\mathbb{R}} \int_{\mathbb{R}} h(y) w\left|v_{0}(s, \xi)\right|^{2} \mathrm{~d} y \mathrm{~d} \xi \mathrm{d} s \\
& \quad \leqslant \epsilon \int_{0}^{\mathrm{t}}\|\sqrt{\mathcal{w} v}\|_{\mathrm{L}^{2}}^{2} \mathrm{~d} s+\frac{\mathrm{C}}{4 \epsilon} \int_{-\tau}^{0}\left\|\sqrt{\mathcal{w}} v_{0}(s)\right\|_{\mathrm{L}^{2}}^{2} \mathrm{ds} .
\end{aligned}
$$

Substituting (3.3) into (3.2), taking $m_{1}>\epsilon$, we get

$$
\|\sqrt{w} \mathcal{v}(\mathrm{t})\|_{\mathrm{L}^{2}}^{2}+\int_{0}^{\mathrm{t}}\|\sqrt{\mathcal{w}} \mathcal{v}(\mathrm{s})\|_{\mathrm{L}^{2}}^{2} \mathrm{~d} \xi \mathrm{d} s \leqslant \mathrm{C}\left\|\sqrt{\mathcal{w}} v_{0}(0)\right\|_{\mathrm{L}^{2}}^{2}+\mathrm{C} \int_{-\tau}^{0}\left\|\sqrt{w} v_{0}(\mathrm{~s})\right\|_{\mathrm{L}^{2}}^{2} \mathrm{~d} s<\infty, \text { for } \mathrm{t} \in[0, \tau] .
$$

On the other hand, we multiply $(2.1)$ by $w(\xi) v(t, \xi)$, and integrate it with respect to $\xi$ and $t$ over $\mathbb{R} \times[0, t]$, but using Cauchy-Schwarz inequality in a different form

$$
\left|\mathrm{D} w^{\prime} v v_{\xi}\right| \leqslant \frac{\mathrm{D}}{2} w v_{\xi}^{2}+\frac{\mathrm{D}}{2}\left(\frac{w^{\prime}}{w}\right)^{2} w v^{2}
$$


we have

$$
\begin{aligned}
& \|\sqrt{w} v(t)\|_{L^{2}}^{2}+D \int_{0}^{t}\left\|\sqrt{w} v \xi_{\xi}(s)\right\|_{L^{2}}^{2} d s \\
& \leqslant 2 m_{2} \int_{0}^{t}\|\sqrt{w} v(s)\|_{L^{2}}^{2} d s+2 \int_{0}^{t} \int_{\mathbb{R}} \int_{\mathbb{R}} h(y) P\left(v_{0}\left(s-\tau, \xi-y-c_{*} \tau\right)\right) w v d y d \xi d s+\left\|\sqrt{w} v_{0}(0)\right\|_{L^{2}}^{2},
\end{aligned}
$$

where $m_{2}=\left|2 D \lambda_{*}^{2}-c_{*} \lambda_{*}+d\right|$. According to (3.4), we can get the estimate for $\int_{0}^{t}\left\|\sqrt{w} v_{\xi}\right\|_{L^{2}}^{2} d s$ :

$$
\|\sqrt{w} v(t)\|_{L^{2}}^{2}+\int_{0}^{t}\left\|\sqrt{w} v_{\xi}(s)\right\|_{L^{2}}^{2} d s \leqslant C\left\|\sqrt{w} v_{0}(0)\right\|_{L^{2}}^{2}+C \int_{-\tau}^{0}\left\|\sqrt{w} v_{0}(s)\right\|_{L^{2}}^{2} d s<\infty, \text { for } t \in[0, \tau] .
$$

Similarly, differentiating (2.1) with respect to $\xi$ and multiplying it by $w(\xi) v_{\xi}(t, \xi)$, and integrating the resultant equation over $[0, t] \times \mathbb{R}$ for $t \in[0, \tau]$, we can prove

$$
\left\|\sqrt{w} v_{\xi}(t)\right\|_{L^{2}}^{2}+\int_{0}^{t}\|\sqrt{w} v \xi \xi(s)\|_{L^{2}}^{2} d s \leqslant C\left\|\sqrt{w} v_{0, \xi}(0)\right\|_{L^{2}}^{2}+C \int_{-\tau}^{0}\left\|\sqrt{w} v_{0}(s)\right\|_{H^{1}}^{2} d s<\infty, \text { for } t \in[0, \tau] .
$$

From (3.1), we have

$$
\begin{aligned}
\|v(t)\|_{C} & \leqslant e^{-d t}\left\|v_{0}(0)\right\|_{C}+C \sup _{s-\tau \in[-\tau, 0]}\left\|v_{0}(s-\tau)\right\|_{C} \int_{0}^{t} e^{-d(t-s)} d s \\
& \leqslant e^{-d t}\left\|v_{0}(0)\right\|_{C}+C \sup _{t-\tau \in[-\tau, 0]}\left\|v_{0}(t-\tau)\right\|_{C} \\
& <\infty \text { for } t \in[0, \tau] .
\end{aligned}
$$

On the other hand, since $v_{0} \in C_{\text {unif }}(-\tau, 0)$, namely, $\lim _{\xi \rightarrow \infty} v_{0}(t, \xi)=: v_{0, \infty}(t) \in C[-\tau, 0]$ and $\lim _{\xi \rightarrow \infty} \partial_{\xi}^{k} v_{0}(t, \xi)=0$ all exist uniformly in $t$ for $k=1,2$, we can prove $v \in C_{\text {unif }}[0, \tau]$. In fact,

$$
\begin{aligned}
\lim _{\xi \rightarrow \infty} v(t, \xi)= & e^{-d t} \int_{\mathbb{R}} G(\eta, t) \lim _{\xi \rightarrow \infty} v_{0}(0, \xi-\eta) d \eta+\int_{0}^{t} e^{-d(t-s)} \int_{\mathbb{R}} G(\eta, t-s) \int_{\mathbb{R}} h(y) \\
& \cdot \lim _{\xi \rightarrow \infty} P\left(v_{0}\left(s-\tau, \xi-\eta-y-c_{*} \tau\right)\right) d y d \eta d s \\
= & e^{-d t} v_{0, \infty}(0)+\int_{0}^{t} e^{-d(t-s)} P\left(v_{0, \infty}(s-\tau)\right) d s \\
:= & g_{1}(t), \quad \text { uniformly with respect to } t \in[0, \tau] .
\end{aligned}
$$

Similarly, noting the facts

$$
\left.G(\eta, t)\right|_{\eta= \pm \infty}=0 \text { and }\left.\left(\partial_{\eta} G(\eta, t)\right)\right|_{\eta= \pm \infty}=0,
$$

we can prove that, for $k=1,2$,

$$
\begin{aligned}
\lim _{\xi \rightarrow \infty} \partial_{\xi}^{k} v(t, \xi)= & e^{-d t} \int_{\mathbb{R}} \partial_{\eta}^{k} G(\eta, t) \lim _{\xi \rightarrow \infty} v_{0}(0, \xi-\eta) d \eta+\int_{0}^{t} e^{-d(t-s)} \int_{\mathbb{R}} \partial_{\eta}^{k} G(\eta, t-s) \\
& \cdot \int_{\mathbb{R}} h(y) \lim _{\xi \rightarrow \infty} P\left(v_{0}\left(s-\tau, \xi-\eta-y-c_{*} \tau\right)\right) d y d \eta d s \\
= & e^{-d t} v_{0, \infty}(0) \int_{\mathbb{R}} \partial_{\eta}^{k} G(\eta, t) d \eta+\int_{0}^{t} e^{-d(t-s)} P\left(v_{0, \infty}(s-\tau)\right) \\
& \cdot \int_{\mathbb{R}} \partial_{\eta}^{k} G(\eta, t-s) \int_{\mathbb{R}} h(y) d y d \eta d s \\
= & 0, \quad \text { uniformly with respect to } t \in[0, \tau] .
\end{aligned}
$$


Thus, (3.4)-(3.10) imply $v \in X_{\mathrm{loc}}(0, \tau)$, and

$$
\begin{aligned}
& \|v(t)\|_{C}^{2}+\|\sqrt{w} v(t)\|_{\mathrm{H}^{1}}^{2}+\int_{0}^{t}\|\sqrt{w} v(s)\|_{\mathrm{H}^{2}}^{2} \mathrm{ds} \\
& \quad \leqslant \mathrm{C}\left(\left\|v_{0}(0)\right\|_{\mathrm{C}}^{2}+\left\|\sqrt{w} v_{0}(0)\right\|_{\mathrm{H}^{1}}^{2}+\int_{-\tau}^{0}\left\|\sqrt{w} v_{0}(s)\right\|_{\mathrm{H}^{2}}^{2} \mathrm{ds}\right), \quad \mathrm{t} \in[0, \tau]
\end{aligned}
$$

for some $\mathrm{C}>1$.

When $t \in[\tau, 2 \tau],(2.1)$ with the initial data $v(s, \xi)$ for $s \in[0, \tau]$ is still linear because the source term $\mathrm{P}_{1}\left(v\left(t-\tau, \xi-y-c_{*} \tau\right)\right)$ is known since $t-\tau \in[0, \tau]$ and $v\left(t-\tau, \xi-y-c_{*} \tau\right)$ is solved in (3.1). So the solution $v(t, \xi)$ for $t \in[\tau, 2 \tau]$ is uniquely and explicitly given by,

$$
\begin{aligned}
v(t, \xi)= & e^{-d t} \int_{\mathbb{R}} G(\eta, t) v(\tau, \xi-\eta) d \eta+\int_{\tau}^{t} e^{-d(t-s)} \int_{\mathbb{R}} G(\eta, t-s) \\
& \cdot \int_{\mathbb{R}} h(y) P\left(v\left(s-\tau, \xi-\eta-y-c_{*} \tau\right)\right) d y d \eta d s .
\end{aligned}
$$

By taking the same estimates as in (3.4)-(3.10), we can prove $v \in X_{\text {loc }}(\tau, 2 \tau)$, that is,

$$
\begin{aligned}
& \|v(t)\|_{C}^{2}+\|\sqrt{w} v(s)\|_{\mathrm{H}^{1}}^{2}+\int_{\tau}^{t}\|\sqrt{w} v(s)\|_{\mathrm{H}^{2}}^{2} \mathrm{ds} \\
& \leqslant \mathrm{C}\left(\|v(\tau)\|_{\mathrm{C}}^{2}+\|\sqrt{w} v(\tau)\|_{\mathrm{H}^{1}}^{2}+\int_{0}^{\tau}\|\sqrt{w} \mathcal{v}(s)\|_{\mathrm{H}^{2}}^{2} \mathrm{ds}\right) \\
& \leqslant \mathrm{C}^{2}\left(\left\|v_{0}(0)\right\|_{\mathrm{C}}^{2}+\left\|\sqrt{w} v_{0}(0)\right\|_{\mathrm{H}^{1}}^{2}+\int_{-\tau}^{0}\left\|\sqrt{w} v_{0}(s)\right\|_{\mathrm{H}^{2}}^{2} \mathrm{ds}\right), \mathrm{t} \in[\tau, 2 \tau] .
\end{aligned}
$$

As similarly shown in (3.9) and (3.10) that

$$
\begin{aligned}
\lim _{\xi \rightarrow \infty} v(t, \xi)= & e^{-d t} \int_{\mathbb{R}} G(\eta, t) \lim _{\xi \rightarrow \infty} v(\tau, \xi-\eta) d \eta+\int_{\tau}^{t} e^{-d(t-s)} \int_{\mathbb{R}} G(\eta, t-s) \int_{\mathbb{R}} h(y) \\
& \cdot \lim _{\xi \rightarrow+\infty} P\left(v\left(s-\tau, \xi-\eta-y-c_{*} \tau\right)\right) d y d \eta d s \\
= & e^{-d t} g_{1}(\tau)+\int_{\tau}^{t} e^{-d(t-s)} P\left(g_{1}(s-\tau)\right) d s \\
:= & g_{2}(t), \quad \text { uniformly with respect to } t \in[0, \tau],
\end{aligned}
$$

and

$$
\begin{aligned}
\lim _{\xi \rightarrow \infty} \partial_{\xi}^{k} v(t, \xi)= & e^{-d t} \int_{\mathbb{R}} \partial_{\eta}^{k} G(\eta, t) \lim _{\xi \rightarrow \infty} v(\tau, \xi-\eta) d \eta+\int_{\tau}^{t} e^{-d(t-s)} \int_{\mathbb{R}} \partial_{\eta}^{k} G(\eta, t-s) \\
& \cdot \int_{\mathbb{R}} h(y) \lim _{\xi \rightarrow \infty} P\left(v\left(s-\tau, \xi-y-c_{*} \tau\right)\right) d y d \eta d s \\
= & g_{1}(\tau) e^{-d t} \int_{\mathbb{R}} \partial_{\eta}^{k} G(\eta, t) d \eta+\int_{\tau}^{t} e^{-d(t-s)} P\left(g_{1}(s-\tau)\right) \int_{\mathbb{R}} \partial_{\eta}^{k} G(\eta, t-s) \cdot \int_{\mathbb{R}} h(y) d y d \eta d s \\
= & 0, \quad \text { uniformly with respect to } t \in[\tau, 2 \tau] .
\end{aligned}
$$

Repeating the above steps, we can prove that $v \in X_{\text {loc }}((n-1) \tau, n \tau)$, uniquely exists, and satisfies

$$
\begin{aligned}
& \|v(t)\|_{C}^{2}+\|\sqrt{w} v(t)\|_{H^{1}}^{2}+\int_{(n-1) \tau}^{t}\|\sqrt{w} v(s)\|_{H^{2}}^{2} d s \\
& \leqslant C^{n}\left(\left\|v_{0}(0)\right\|_{C}^{2}+\left\|\sqrt{w} v_{0}(0)\right\|_{H^{1}}^{2}+\int_{-\tau}^{0}\left\|\sqrt{w} v_{0}(s)\right\|_{H^{2}}^{2} d s\right), t \in[(n-1) \tau, n \tau] .
\end{aligned}
$$


And finally we prove that $v$ is unique, and $v \in \mathrm{X}_{\mathrm{loc}}(0, \infty)$ with, for any $\mathrm{T}>0$, that

$$
\begin{aligned}
& \|v(t)\|_{C}^{2}+\|\sqrt{w} v(t)\|_{\mathrm{H}^{1}}^{2}+\int_{0}^{t}\|\sqrt{w} v(s)\|_{\mathrm{H}^{2}}^{2} \mathrm{ds} \\
& \leqslant \mathrm{C}_{\mathrm{T}}\left(\left\|v_{0}(0)\right\|_{\mathrm{C}}^{2}+\left\|\sqrt{w} v_{0}(0)\right\|_{\mathrm{H}^{1}}^{2}+\int_{-\tau}^{0}\left\|\sqrt{w} v_{0}(s)\right\|_{\mathrm{H}^{2}}^{2} \mathrm{~d} s\right), \mathrm{t} \in[0, \mathrm{~T}] .
\end{aligned}
$$

\section{Proof of uniform boundedness}

To our knowledge, the weighted-energy method in $[6,10]$ can not estimate the boundedness of $\int_{0}^{\infty}\left\|\sqrt{w} v_{\xi}\right\|_{L^{2}}^{2}$, since we can not use the Cauchy-Schwarz inequality to control the $v v_{\xi}$ by $v_{\xi}^{2}$. Thus, we must find a different method to handle it. Here we adopt the so-called anti-weighted method [5, 8, 15], namely, let $v(t, \xi)=\frac{1}{\sqrt{w(\xi)}} \widetilde{v}(t, \xi)$, i.e., $\widetilde{v}(t, \xi)=\sqrt{w(\xi)} v(t, \xi)=e^{-\lambda_{*} \xi} v(t, \xi)$.

We obtain the following equations for the new known $\widetilde{v}(t, \xi)$ satisfies

$$
\begin{gathered}
\widetilde{v}_{t}(t, \xi)+k_{1} \widetilde{v}_{\xi}(t, \xi)-D \widetilde{v}_{\xi \xi}(t, \xi)+k_{2} \widetilde{v}(t, \xi)-\int_{\mathbb{R}} h(y) b^{\prime}\left(\phi\left(\xi-y-c_{*} \tau\right)\right) e^{-\lambda_{*}\left(y+c_{*} \tau\right)} \\
\cdot \widetilde{v}\left(t-\tau, \xi-y-c_{*} \tau\right) d y=\int_{\mathbb{R}} h(y) \widetilde{Q}\left(\widetilde{v}\left(t-\tau, \xi-y-c_{*} \tau\right)\right) d y, \\
\widetilde{v}(s, \xi)=\sqrt{w(\xi)} v(s, \xi)=\widetilde{v}_{0}(s, \xi), \quad(s, \xi) \in[-\tau, 0] \times \mathbb{R},
\end{gathered}
$$

where

$$
k_{1}=C_{*}-2 D \lambda_{*}, \quad k_{2}=c_{*} \lambda_{*}-D \lambda_{*}^{2}+d
$$

satisfying

$$
k_{2}=c_{*} \lambda_{*}-D \lambda_{*}^{2}+d=b^{\prime}(0) \int_{\mathbb{R}} h(y) e^{-\lambda_{*}\left(y+c_{*} \tau\right)} d y
$$

and

$$
\widetilde{\mathrm{Q}}(\widetilde{v})=e^{-\lambda_{*} \xi} \mathrm{Q}(v)
$$

satisfying

$$
|\widetilde{\mathrm{Q}}(\widetilde{v})| \leqslant C e^{-\lambda_{*} \xi}|v|^{2}=\frac{\mathrm{C}}{\sqrt{w(\xi)}}|\widetilde{v}|^{2}
$$

where $v=v\left(t-\tau, \xi-y-c_{*} \tau\right)$.

Now we establish the uniform boundedness of the solution $v \in X(0, \infty)$ by several lemmas.

Lemma 4.1. It holds that

$$
\begin{aligned}
& \|\widetilde{v}(t)\|_{L^{2}}^{2}+\int_{0}^{t} \int_{\mathbb{R}} A(\xi)|\widetilde{v}(s, \xi)|^{2} d \xi d s+2 D \int_{0}^{t}\left\|\widetilde{v}_{\xi}(s)\right\|_{L^{2}}^{2} d s \\
& \leqslant\left\|\widetilde{v}_{0}(0)\right\|_{\mathrm{L}^{2}}^{2}+\mathrm{C} \int_{0}^{\mathrm{t}} \int_{\mathbb{R}} \int_{\mathbb{R}} \mathrm{h}(\mathrm{y}) \frac{1}{\sqrt{\mathcal{w}(\xi)}}|\widetilde{v}(s, \xi)|\left|\widetilde{v}\left(s-\tau, \xi-y-c_{*} \tau\right)\right|^{2} \mathrm{~d} y \mathrm{~d} \xi \mathrm{d} s \\
& +\mathrm{b}^{\prime}(0) \int_{-\tau}^{0} \int_{\mathbb{R}} \int_{\mathbb{R}} e^{-\lambda_{*}\left(y+c_{*} \tau\right)}\left|\widetilde{v}_{0}(s, \xi)\right|^{2} d y d \xi d s,
\end{aligned}
$$

where

$$
A(\xi):=\int_{\mathbb{R}} h(y) e^{-\lambda_{*}\left(y+c_{*} \tau\right)}\left[2 b^{\prime}(0)-\left|b^{\prime}(\phi(\xi))\right|-\left|b^{\prime}\left(\phi\left(\xi-y-c_{*} \tau\right)\right)\right|\right] d y
$$


Proof. Multiplying (4.1) by $\widetilde{v}$ and integrating it with respect to $\xi$ and $t$ over $\mathbb{R} \times[0, t]$, we have

$$
\begin{aligned}
& \|\widetilde{v}(t)\|_{L^{2}}^{2}+2 k_{2} \int_{0}^{t} \int_{\mathbb{R}}|\widetilde{v}(s, \xi)|^{2} \mathrm{~d} \xi \mathrm{d} s+2 \mathrm{D} \int_{0}^{\mathrm{t}}\left\|\widetilde{v}_{\xi}(\mathrm{s})\right\|_{\mathrm{L}^{2}}^{2} \mathrm{~d} s \\
& -2 \int_{0}^{t} \int_{\mathbb{R}} \int_{\mathbb{R}} h(y) b^{\prime}\left(\phi\left(\xi-y-c_{*} \tau\right)\right) e^{-\lambda_{*}\left(y+c_{*} \tau\right)} \widetilde{\mathcal{V}}\left(s-\tau, \xi-y-c_{*} \tau\right) d y d \xi d s \\
& =2 \int_{0}^{t} \int_{\mathbb{R}} \int_{\mathbb{R}} h(y) \widetilde{Q}\left(\widetilde{v}\left(t-\tau, \xi-y-c_{*} \tau\right)\right) \widetilde{v} d y d \xi d s+\left\|\widetilde{v}_{0}(0)\right\|_{L^{2}}^{2} .
\end{aligned}
$$

Using the Cauchy-Schwarz inequality and the change of variables, we can estimate

$$
\begin{aligned}
& \left|2 \int_{0}^{t} \int_{\mathbb{R}} \int_{\mathbb{R}} h(y) b^{\prime}\left(\phi\left(\xi-y-c_{*} \tau\right)\right) e^{-\lambda_{*}\left(y+c_{*} \tau\right)} \widetilde{\mathcal{v}}\left(s-\tau, \xi-y-c_{*} \tau\right) d y d \xi d s\right| \\
& \leqslant \int_{0}^{t} \int_{\mathbb{R}} \int_{\mathbb{R}} h(y)\left|b^{\prime}\left(\phi\left(\xi-y-c_{*} \tau\right)\right)\right| e^{-\lambda_{*}\left(y+c_{*} \tau\right)}|\widetilde{v}(s, \xi)|^{2} d y d \xi d s \mid \\
& +\int_{0}^{t} \int_{\mathbb{R}} \int_{\mathbb{R}} h(y)\left|b^{\prime}\left(\phi\left(\xi-y-c_{*} \tau\right)\right)\right| e^{-\lambda_{*}\left(y+c_{*} \tau\right)}\left|\widetilde{v}\left(s-\tau, \xi-y-c_{*} \tau\right)\right|^{2} d y d \xi d s \\
& =\int_{0}^{t} \int_{\mathbb{R}} \int_{\mathbb{R}} h(y)\left|b^{\prime}\left(\phi\left(\xi-y-c_{*} \tau\right)\right)\right| e^{-\lambda_{*}\left(y+c_{*} \tau\right)}|\widetilde{v}(s, \xi)|^{2} d y d \xi d s \\
& +\int_{-\tau}^{t-\tau} \int_{\mathbb{R}} \int_{\mathbb{R}} h(y)\left|b^{\prime}(\phi(\xi))\right| e^{-\lambda_{*}\left(y+c_{*} \tau\right)}|\widetilde{v}(s, \xi)|^{2} d y d \xi d s \\
& \leqslant \int_{0}^{t} \int_{\mathbb{R}} \int_{\mathbb{R}} \mathrm{h}(\mathrm{y})\left[\left|\mathrm{b}^{\prime}\left(\phi\left(\xi-\mathrm{y}-\mathrm{c}_{*} \tau\right)\right)\right|+\left|\mathrm{b}^{\prime}(\phi(\xi))\right|\right] e^{-\lambda_{*}\left(y+c_{*} \tau\right)}|\widetilde{v}(s, \xi)|^{2} \mathrm{~d} y \mathrm{~d} \xi \mathrm{d} s \\
& +\mathrm{b}^{\prime}(0) \int_{-\tau}^{0} \int_{\mathbb{R}} \int_{\mathbb{R}} h(y) e^{-\lambda_{*}\left(y+c_{*} \tau\right)}\left|\widetilde{v}_{0}(s, \xi)\right|^{2} \mathrm{~d} y \mathrm{~d} \xi \mathrm{d} s .
\end{aligned}
$$

On the other hand, we have

$$
\begin{aligned}
& \left|\int_{0}^{t} \int_{\mathbb{R}} \int_{\mathbb{R}} h(y) \widetilde{Q}\left(\widetilde{v}\left(t-\tau, \xi-y-c_{*} \tau\right)\right) \widetilde{v} d y d \xi d s\right| \\
& \quad \leqslant\left. C \int_{0}^{t} \int_{\mathbb{R}} \int_{\mathbb{R}} h(y) \frac{1}{\sqrt{w(\xi)}}|\widetilde{v}| \widetilde{v}\left(s-\tau, \xi-y-c_{*} \tau\right)\right|^{2} d y d \xi d s .
\end{aligned}
$$

Combining (4.2), (4.3), and (4.4), we get

$$
\begin{aligned}
& \|\widetilde{v}(\mathrm{t})\|_{\mathrm{L}^{2}}^{2}+\int_{0}^{\mathrm{t}} \int_{\mathbb{R}} \mathrm{A}(\xi)|\widetilde{v}(\mathrm{~s}, \xi)|^{2} \mathrm{~d} \xi \mathrm{d} s+2 \mathrm{D} \int_{0}^{\mathrm{t}}\left\|\widetilde{v}_{\xi}(\mathrm{s})\right\|_{\mathrm{L}^{2}}^{2} \mathrm{~d} s \\
& \leqslant\left\|\widetilde{v}_{0}(0)\right\|_{\mathrm{L}^{2}}^{2}+\mathrm{C} \int_{0}^{\mathrm{t}} \int_{\mathbb{R}} \int_{\mathbb{R}} h(\mathrm{y}) \frac{1}{\sqrt{w(\xi)}}\left|\widetilde{v}(s, \xi) \| \widetilde{v}\left(s-\tau, \xi-y-\mathrm{c}_{*} \tau\right)\right|^{2} \mathrm{~d} y \mathrm{~d} \xi \mathrm{d} s \\
& \quad+\mathrm{b}^{\prime}(0) \int_{-\tau}^{0} \int_{\mathbb{R}} \int_{\mathbb{R}} e^{-\lambda_{*}\left(y+\mathrm{c}_{*} \tau\right)}\left|\widetilde{v}_{0}(s, \xi)\right|^{2} \mathrm{~d} y \mathrm{~d} \xi \mathrm{d} s,
\end{aligned}
$$

where

$$
\begin{aligned}
A(\xi) & \left.:=2 k_{2}-\int_{\mathbb{R}} h(y)\left[\left|b^{\prime}\left(\phi\left(\xi-y-c_{*} \tau\right)\right)\right|+\left|b^{\prime}(\phi(\xi))\right|\right] e^{-\lambda_{*}\left(y+c_{*} \tau\right)}\right] d y \\
& =2\left[c_{*} \lambda_{*}-D \lambda_{*}^{2}+d\right]-\int_{\mathbb{R}} h(y)\left[\left|b^{\prime}\left(\phi\left(\xi-y-c_{*} \tau\right)\right)\right|+\left|b^{\prime}(\phi(\xi))\right|\right] e^{-\lambda_{*}\left(y+c_{*} \tau\right)} d y \\
& =\int_{\mathbb{R}} h(y) e^{-\lambda_{*}\left(y+c_{*} \tau\right)}\left[2 b^{\prime}(0)-\left|b^{\prime}(\phi(\xi))\right|-\left|b^{\prime}\left(\phi\left(\xi-y-c_{*} \tau\right)\right)\right|\right] d y .
\end{aligned}
$$

Lemma 4.2. It holds that

$$
A(\xi) \geqslant C_{2} \phi(\xi)>0
$$

for some positive constant $\mathrm{C}_{2}$. 
Proof. The proof is similar to [1], so we omit it.

Lemma 4.3. There exists $\delta_{1}>0$, when $M_{\infty} \leqslant \delta_{1}$, then

$$
\begin{aligned}
& \|\widetilde{v}(\mathrm{t})\|_{\mathrm{L}^{2}}^{2}+\int_{0}^{\mathrm{t}} \int_{\mathbb{R}} \phi(\xi) w(\xi)|v(s, \xi)|^{2} \mathrm{~d} \xi \mathrm{d} s+\int_{0}^{\mathrm{t}}\left\|\widetilde{v}_{\xi}(\mathrm{s})\right\|_{\mathrm{L}^{2}}^{2} \mathrm{~d} s \\
& \quad \leqslant \mathrm{C}_{3}\left(\left\|\widetilde{v}_{0}(0)\right\|_{\mathrm{L}^{2}}^{2}+\int_{-\tau}^{0}\left\|\widetilde{v}_{0}(\mathrm{~s})\right\|_{\mathrm{L}^{2}}^{2} \mathrm{~d} s\right) \leqslant \mathrm{C}_{3} \mathrm{M}_{0}^{2}, \mathrm{t} \in[0, \infty),
\end{aligned}
$$

where $\mathrm{C}_{3}$ is a positive constant.

Proof. Since $\widetilde{v}(t, \xi)=\sqrt{w(\xi)} v(t, \xi)$, by lemma 4.2, then the second term of the left-hand-side of (4.2) can be written as

$$
\int_{0}^{t} \int_{\mathbb{R}} A(\xi)|\widetilde{v}(s, \xi)|^{2} \mathrm{~d} \xi \mathrm{d} s \geqslant \mathrm{C}_{2} \int_{0}^{\mathrm{t}} \int_{\mathbb{R}} \phi(\xi) w(\xi)|v(s, \xi)|^{2} \mathrm{~d} \xi \mathrm{d} s .
$$

This can be used to control the nonlinear term in (4.2).

Now we are going to estimate the nonlinear term in (4.2). It can be reformed as

$$
\begin{aligned}
& \int_{0}^{t} \int_{\mathbb{R}} \int_{\mathbb{R}} h(y) \frac{1}{\sqrt{w(\xi)}}|\widetilde{v}(s, \xi)|\left|\widetilde{v}\left(s-\tau, \xi-y-c_{*} \tau\right)\right|^{2} d y d \xi d s \\
& \quad=\int_{0}^{t} \int_{\mathbb{R}} \int_{\mathbb{R}} h(y) w\left(\xi-y-c_{*} \tau\right)|v(s, \xi)|\left|v\left(s-\tau, \xi-y-c_{*} \tau\right)\right|^{2} d y d \xi d s \\
& \quad=\int_{0}^{t} \int_{\mathbb{R}} \int_{\mathbb{R}} h(y) \phi\left(\xi-y-c_{*} \tau\right) w\left(\xi-y-c_{*} \tau\right) \frac{|v(s, \xi)|}{\phi\left(\xi-y-c_{*} \tau\right)}\left|v\left(s-\tau, \xi-y-c_{*} \tau\right)\right|^{2} d y d \xi d s .
\end{aligned}
$$

So we need further to estimate $\frac{|v(s, \xi)|}{\phi\left(\xi-y-c_{*} \tau\right)}$.

Notice that the critical wave $\phi\left(x+c_{*} t\right)$ is positive and bounded, and $\lim _{\xi \rightarrow+\infty} \phi(\xi)=u_{+}$and $\phi(\xi)=$ $\mathrm{O}(1)|\xi| e^{\lambda_{*} \xi} \rightarrow 0$ as $\xi \rightarrow-\infty$, so, there exists a number $\xi_{1}$ near $-\infty$, i.e., $\xi_{1}<0$ and $\left|\xi_{1}\right| \gg 1$, such that $\phi(\xi)=\mathrm{O}(1)|\xi| e^{\lambda_{*}} \xi \rightarrow 0$ for $\xi \in\left(-\infty, \xi_{1}\right)$, and $\phi(\xi)=\mathrm{O}(1)$ for $\xi \in\left[\xi_{1}, \infty\right)$. Thus, by the definition of $w(\xi)$, we can verify that

$$
\frac{1}{\phi\left(\xi-y-c_{*} \tau\right)} \leqslant \begin{cases}C \sqrt{w(\xi)}, & t \in\left(-\infty, \xi_{1}\right), \\ C, & t \in\left[\xi_{1}, \infty\right)\end{cases}
$$

for some positive constant $C$. This with the definition of solution space $X(-\tau, \infty)$ and the definition of $M_{\infty}$ as well as Sobolev inequality guarantees

$$
\begin{aligned}
\sup _{\xi \in \mathbb{R}} \frac{|v(t, \xi)|}{\phi\left(\xi-y-c_{*} \tau\right)} & \leqslant \sup _{\xi \in\left(-\infty, \xi_{1}\right)} C \sqrt{w}|v(t)|+\sup _{\xi \in\left[\xi_{1}, \infty\right)} C|v(t)| \\
& \leqslant C \sup _{\xi \in \mathbb{R}} \sqrt{w}|v(t)|+C \sup _{\xi \in \mathbb{R}}|v(t)| \leqslant C\|\sqrt{w} v(t)\|_{H^{1}}+C\|v(t)\|_{C} \leqslant C M_{\infty} .
\end{aligned}
$$

Thus, applying the above estimates (4.8), from (4.7) we can estimate the nonlinear term in (4.2) as follows

$$
\begin{aligned}
& \left.\int_{0}^{t} \int_{\mathbb{R}} \int_{\mathbb{R}} h(y) \frac{1}{\sqrt{w(\xi)}} \widetilde{v}(s, \xi)|| \widetilde{v}\left(s-\tau, \xi-y-c_{*} \tau\right)\right|^{2} d y d \xi d s \\
& =\int_{0}^{t} \int_{\mathbb{R}} \int_{\mathbb{R}} h(y) \phi\left(\xi-y-c_{*} \tau\right) w\left(\xi-y-c_{*} \tau\right) \frac{|v(s, \xi)|}{\phi\left(\xi-y-c_{*} \tau\right)}\left|v\left(s-\tau, \xi-y-c_{*} \tau\right)\right|^{2} d y d \xi d s \\
& \leqslant \mathrm{CM}_{\infty} \int_{0}^{\mathrm{t}} \int_{\mathbb{R}} \int_{\mathbb{R}} \mathrm{h}(\mathrm{y}) \phi\left(\xi-y-c_{*} \tau\right) w\left(\xi-y-c_{*} \tau\right)\left|v\left(s-\tau, \xi-y-c_{*} \tau\right)\right|^{2} \mathrm{~d} y \mathrm{~d} \xi \mathrm{d} s \\
& =\mathrm{CM}_{\infty} \int_{-\tau}^{\mathrm{t}-\tau} \int_{\mathbb{R}} \int_{\mathbb{R}} \mathrm{h}(\mathrm{y}) \phi(\xi) w(\xi)|v(s, \xi)|^{2} \mathrm{~d} y \mathrm{~d} \xi \mathrm{d} s \\
& \leqslant \mathrm{CM}_{\infty} \int_{0}^{\mathrm{t}} \int_{\mathbb{R}} \phi(\xi) w(\xi)|v(s, \xi)|^{2} \mathrm{~d} \xi \mathrm{d} s+\mathrm{CM}_{\infty} \int_{-\tau}^{0} \int_{\mathbb{R}} \phi(\xi) w(\xi)\left|v_{0}(s, \xi)\right|^{2} \mathrm{~d} \xi \mathrm{d} s \\
& \leqslant \mathrm{CM}_{\infty} \int_{0}^{\mathrm{t}} \int_{\mathbb{R}} \phi(\xi) \mathcal{w}(\xi)|v(\mathrm{~s}, \xi)|^{2} \mathrm{~d} \xi \mathrm{d} s+\mathrm{C} \int_{-\tau}^{0}\left\|\widetilde{v}_{0}(\mathrm{~s}, \xi)\right\|_{\mathrm{L}^{2}}^{2} \mathrm{~d} s .
\end{aligned}
$$


Combining (4.2), (4.6), and (4.9), we get

$$
\begin{aligned}
& \|\widetilde{v}(\mathrm{t})\|_{\mathrm{L}^{2}}^{2}+\left[\mathrm{C}_{2}-\mathrm{C}_{4} \mathrm{M}_{\infty}\right] \int_{0}^{\mathrm{t}} \int_{\mathbb{R}} \phi(\xi) w(\xi)|v(s, \xi)|^{2} \mathrm{~d} \xi \mathrm{d} s+\int_{0}^{\mathrm{t}}\left\|\widetilde{v}_{\xi}(\mathrm{s})\right\|_{\mathrm{L}^{2}}^{2} \mathrm{~d} s \\
& \quad \leqslant \mathrm{C}_{5}\left(\left\|\widetilde{v}_{0}(0)\right\|_{\mathrm{L}^{2}}^{2}+\int_{-\tau}^{0}\left\|\widetilde{v}_{0}(\mathrm{~s})\right\|_{\mathrm{L}^{2}}^{2} \mathrm{ds}\right)
\end{aligned}
$$

for some positive constants $C_{4}$ and $C_{5}$, which immediately implies (4.5) by taking $M_{\infty}$ to be small, for example, let

$$
0<M_{\infty} \leqslant \delta_{1}:=\frac{C_{2}}{2 C_{4}}
$$

then the corresponding constant $C_{3}$ in (4.5) is

$$
\mathrm{C}_{3}=\frac{\mathrm{C}_{5}}{\min \left\{1, \frac{\mathrm{C}_{2}}{2}\right\}}
$$

The proof is complete.

Similarly, the estimate for $\widetilde{v}_{\xi}$ can be established as follows.

Lemma 4.4. When $\mathrm{M}_{\infty} \leqslant \delta_{1}$, then

$$
\left\|\widetilde{v}_{\xi}(\mathrm{t})\right\|_{\mathrm{L}^{2}}^{2}+\int_{0}^{\mathrm{t}}\left\|\widetilde{v}_{\xi \xi}(\mathrm{s})\right\|_{\mathrm{L}^{2}}^{2} \mathrm{ds} \leqslant \mathrm{C}_{6}\left(\mathrm{M}_{\infty}+1\right) \mathrm{M}_{0}^{2}, \mathrm{t} \in[0, \infty)
$$

and

$$
\int_{0}^{\mathrm{t}}\left|\frac{\mathrm{d}}{\mathrm{ds}}\left\|\widetilde{\nu}_{\xi}(\mathrm{s})\right\|_{\mathrm{L}^{2}}^{2}\right| \mathrm{d} s \leqslant \mathrm{C}_{7}\left(\mathrm{M}_{\infty}+1\right) \mathrm{M}_{0}^{2}, \mathrm{t} \in[0, \infty),
$$

where $\mathrm{C}_{6}$ and $\mathrm{C}_{7}$ are some positive constants.

Proof. Differentiating (4.1) with respect to $\xi$ and multiplying the resultant equation by $\widetilde{v}_{\xi}$ and integrating it with respect to $\xi$ over $\mathbb{R}$, we obtain

$$
\begin{aligned}
& \frac{d}{d t}\left\|\widetilde{v}_{\xi}(t)\right\|_{L^{2}}^{2}+2 k_{2}\left\|\widetilde{v}_{\xi}(t)\right\|_{L^{2}}^{2}+2 D\left\|\widetilde{v}_{\xi \xi}(t)\right\|_{L^{2}}^{2} \\
& =2 \int_{\mathbb{R}} \int_{\mathbb{R}} h(y) b^{\prime}\left(\phi\left(\xi-y-c_{*} \tau\right)\right) e^{-\lambda_{*}\left(y+c_{*} \tau\right)} \widetilde{v}_{\xi} \widetilde{v}_{\xi}\left(t-\tau, \xi-y-c_{*} \tau\right) d y d \xi \\
& \quad+2 \int_{\mathbb{R}} \int_{\mathbb{R}} h(y) b^{\prime \prime}\left(\phi\left(\xi-y-c_{*} \tau\right)\right) \phi^{\prime}\left(\xi-y-c_{*} \tau\right) e^{-\lambda_{*}\left(y+c_{*} \tau\right)} \\
& \quad \cdot \widetilde{v}_{\xi} \widetilde{v}\left(t-\tau, \xi-y-c_{*} \tau\right) d y d \xi+2 \int_{\mathbb{R}} \int_{\mathbb{R}} h(y) \widetilde{Q}_{\xi}\left(\widetilde{v}\left(t-\tau, \xi-y-c_{*} \tau\right)\right) \widetilde{v}_{\xi} d y d \xi \\
& :=I_{1}(t)+I_{2}(t)+I_{3}(t) .
\end{aligned}
$$

Integrating it with respect to $t$ over $[0, t]$, we get

$$
\left\|\widetilde{v}_{\xi}(t)\right\|_{\mathrm{L}^{2}}^{2}+2 \mathrm{k}_{2} \int_{0}^{\mathrm{t}}\left\|\widetilde{v}_{\xi}(\mathrm{s})\right\|_{\mathrm{L}^{2}}^{2} \mathrm{~d} s+2 \mathrm{D} \int_{0}^{\mathrm{t}}\left\|\widetilde{v}_{\xi \xi}(\mathrm{s})\right\|_{\mathrm{L}^{2}}^{2} \mathrm{ds}=\left\|\widetilde{v}_{0, \xi}(0)\right\|_{\mathrm{L}^{2}}^{2}+\int_{0}^{\mathrm{t}}\left[\mathrm{I}_{1}(\mathrm{~s})+\mathrm{I}_{2}(\mathrm{~s})+\mathrm{I}_{3}(\mathrm{~s})\right] \mathrm{ds} .
$$


By using the estimate (4.5), Cauchy-Schwarz inequality, the change of variables, and the facts that $\left|\phi^{\prime}(\xi)\right| \leqslant$ $\mathrm{C} \phi(\xi)$, and $|v(t, \xi)| \leqslant \mathrm{CM}_{\infty}$ for $(\mathrm{t}, \xi) \in \mathbb{R}_{+} \times \mathbb{R}$, we can similarly estimate the nonlinear terms as

$$
\begin{aligned}
& \int_{0}^{t}\left|I_{1}(s)\right| d s \leqslant C \int_{0}^{t} \int_{\mathbb{R}} \int_{\mathbb{R}} h(y) e^{-\lambda_{*}\left(y+c_{*} \tau\right)}\left[\left|\widetilde{v}_{\xi}\right|^{2}+\left|\widetilde{v}_{\xi}\left(s-\tau, \xi-y-c_{*} \tau\right)\right|^{2}\right] d y d \xi d s \\
& =C \int_{0}^{t}\left\|\widetilde{v}_{\xi}\right\|_{L^{2}}^{2} d s+C \int_{-\tau}^{t-\tau} \int_{\mathbb{R}} \int_{\mathbb{R}} h(y) e^{-\lambda_{*}\left(y+c_{*} \tau\right)}\left|\widetilde{v}_{\xi}(s, \xi)\right|^{2} d y d \xi d s \\
& \leqslant \mathrm{C} \int_{0}^{\mathrm{t}}\left\|\widetilde{v}_{\xi}\right\|_{\mathrm{L}^{2}}^{2} \mathrm{ds}+\mathrm{C} \int_{-\tau}^{0} \|\left.\widetilde{v}_{0, \xi}(\mathrm{s})\right|^{2} \mathrm{ds} \\
& \leqslant \mathrm{C}\left(\left\|\widetilde{v}_{0}(0)\right\|_{\mathrm{L}^{2}}^{2}+\int_{-\tau}^{0}\left\|\widetilde{v}_{0, \xi}(\mathrm{s})\right\|_{\mathrm{L}^{2}}^{2} \mathrm{ds}\right) \leqslant \mathrm{CM}_{0}^{2} \\
& \int_{0}^{t}\left|I_{2}(s)\right| d s \leqslant C \int_{0}^{t} \int_{\mathbb{R}} \int_{\mathbb{R}} h(y) e^{-\lambda_{*}\left(y+c_{*} \tau\right)}\left[\left|\widetilde{v}_{\xi}\right|^{2}+\left|\phi^{\prime}\left(\xi-y-c_{*} \tau\right)\right|^{2}\left|\widetilde{v}\left(s-\tau, \xi-y-c_{*} \tau\right)\right|^{2}\right] d y d \xi d s \\
& =\mathrm{C} \int_{0}^{\mathrm{t}}\left\|\widetilde{v}_{\xi}\right\|_{\mathrm{L}^{2}}^{2} \mathrm{~d} s+\mathrm{C} \int_{0}^{\mathrm{t}} \int_{\mathbb{R}} \int_{\mathbb{R}} \mathrm{h}(\mathrm{y}) e^{-\lambda_{*}\left(y+\boldsymbol{c}_{*} \tau\right)}\left|\phi^{\prime}\left(\xi-\mathrm{y}-\mathrm{c}_{*} \tau\right)\right|^{2} \\
& \cdot\left|\widetilde{v}\left(s-\tau, \xi-y-c_{*} \tau\right)\right|^{2} d y d \xi d s \\
& \leqslant \mathrm{C} \int_{0}^{\mathrm{t}}\left\|\widetilde{v}_{\xi}\right\|_{\mathrm{L}^{2}}^{2} \mathrm{~d} s+\mathrm{C} \int_{0}^{\mathrm{t}} \int_{\mathbb{R}} \int_{\mathbb{R}} \mathrm{h}(\mathrm{y}) \mathrm{e}^{-\lambda_{*}\left(y+\mathrm{c}_{*} \tau\right)} \phi\left(\xi-\mathrm{y}-\mathrm{c}_{*} \tau\right) \\
& \cdot w\left(\xi-y-c_{*} \tau\right)\left|v\left(s-\tau, \xi-y-c_{*} \tau\right)\right|^{2} d y d \xi d s \\
& =\mathrm{C} \int_{0}^{\mathrm{t}}\left\|\widetilde{v}_{\xi}\right\|_{\mathrm{L}^{2}}^{2} \mathrm{ds}+\mathrm{C} \int_{-\tau}^{\mathrm{t}-\tau} \int_{\mathbb{R}} \int_{\mathbb{R}} \mathrm{h}(\mathrm{y}) e^{-\lambda_{*}\left(y+c_{*} \tau\right)} \phi(\xi) w(\xi)|v(s, \xi)|^{2} \mathrm{~d} y \mathrm{~d} \xi \mathrm{d} s \\
& \leqslant C \int_{0}^{t}\left\|\widetilde{v}_{\xi}\right\|_{\mathrm{L}^{2}}^{2} \mathrm{~d} s+\mathrm{C} \int_{-\tau}^{0} \int_{\mathbb{R}} \int_{\mathbb{R}} h(y) e^{-\lambda_{*}\left(y+c_{*} \tau\right)}\left|\widetilde{v}_{0}(s, \xi)\right|^{2} \mathrm{~d} y \mathrm{~d} \xi \mathrm{ds} \\
& +\mathrm{C} \int_{0}^{t} \int_{\mathbb{R}} \int_{\mathbb{R}} h(y) e^{-\lambda_{*}\left(y+c_{*} \tau\right)} \phi(\xi) w(\xi)\left|\widetilde{v}_{0}(s, \xi)\right|^{2} d y d \xi d s \\
& \leqslant \mathrm{C}\left(\left\|\widetilde{v}_{0}(0)\right\|_{\mathrm{L}^{2}}^{2}+\int_{-\tau}^{0}\left\|\widetilde{v}_{0}(\mathrm{~s})\right\|_{\mathrm{L}^{2}}^{2} \mathrm{ds}\right) \leqslant \mathrm{CM}_{0}^{2} \\
& \int_{0}^{t}\left|I_{3}(s)\right| d s \leqslant C \int_{0}^{t} \int_{\mathbb{R}} \int_{\mathbb{R}} \frac{h(y)}{\sqrt{w(\xi)}}\left|\widetilde{v}_{\xi}\right|\left|\widetilde{v}_{\xi}\left(s-\tau, \xi-y-c_{*} \tau\right)\right|\left|\widetilde{v}\left(s-\tau, \xi-y-c_{*} \tau\right)\right| d y d \xi d s \\
& \leqslant \mathrm{CM}_{\infty} \int_{0}^{\mathrm{t}} \int_{\mathbb{R}} \int_{\mathbb{R}} \mathrm{h}(\mathrm{y})\left|\widetilde{v}_{\xi}\right| \widetilde{v}_{\xi}\left(\mathrm{s}-\tau, \xi-\mathrm{y}-\mathrm{c}_{*} \tau\right) \mid \mathrm{d} \mathrm{yd} \xi \mathrm{d} s \\
& \leqslant \mathrm{CM}_{\infty} \int_{0}^{\mathrm{t}} \int_{\mathbb{R}} \int_{\mathbb{R}} \mathrm{h}(\mathrm{y})\left[\left.\widetilde{v}_{\xi}\right|^{2}+\left|\widetilde{v}_{\xi}\left(s-\tau, \xi-y-c_{*} \tau\right)\right|^{2}\right] \mathrm{d} y \mathrm{~d} \xi \mathrm{d} s \\
& =\mathrm{CM}_{\infty} \int_{0}^{\mathrm{t}}\left\|\widetilde{v}_{\xi}\right\|_{\mathrm{L}^{2}}^{2} \mathrm{~d} s+\mathrm{CM}_{\infty} \int_{-\tau}^{\mathrm{t}-\tau} \int_{\mathbb{R}} \int_{\mathbb{R}} h(\mathrm{y})\left|\widetilde{v}_{\xi}(s, \xi)\right|^{2} \mathrm{~d} y \mathrm{~d} \xi \mathrm{d} s \\
& \leqslant \mathrm{CM}_{\infty} \int_{0}^{\mathrm{t}}\left\|\widetilde{v}_{\xi}\right\|_{\mathrm{L}^{2}}^{2} \mathrm{~d} s+\mathrm{CM}_{\infty} \int_{-\tau}^{0} \|\left.\widetilde{v}_{0, \xi}(\mathrm{s})\right|^{2} \mathrm{~d} s \\
& \leqslant \mathrm{CM}_{\infty}\left(\left\|\widetilde{v}_{0}(0)\right\|_{\mathrm{L}^{2}}^{2}+\int_{-\tau}^{0}\left\|\widetilde{v}_{0, \xi}(\mathrm{s})\right\|_{\mathrm{L}^{2}}^{2} \mathrm{ds}\right) \\
& \leqslant \mathrm{CM}_{\infty} \mathrm{M}_{0}^{2}
\end{aligned}
$$

provided $M_{\infty} \leqslant \delta_{1}$ 
Therefore, substituting (4.13)-(4.15) to (4.12), we get

$$
\left\|\widetilde{v}_{\xi}(t)\right\|_{\mathrm{L}^{2}}^{2}+\int_{0}^{\mathrm{t}}\left\|\widetilde{v}_{\xi \xi}(\mathrm{s})\right\|_{\mathrm{L}^{2}}^{2} \mathrm{~d} s \leqslant \mathrm{C}_{6}\left(\mathrm{M}_{\infty}+1\right)\left(\left\|\widetilde{v}_{0}(0)\right\|_{\mathrm{H}^{1}}^{2}+\int_{-\tau}^{0}\left\|\widetilde{v}_{0}(\mathrm{~s})\right\|_{\mathrm{H}^{1}}^{2} \mathrm{ds}\right) \leqslant \mathrm{C}_{6}\left(\mathrm{M}_{\infty}+1\right) \mathrm{M}_{0}^{2}
$$

for some constant $C_{6}>0$, provided $M_{\infty} \leqslant \delta_{1}$. This proves (4.10).

From (4.11), we have

$$
\left|\frac{\mathrm{d}}{\mathrm{dt}}\right|\left|\widetilde { v } _ { \xi } ( \mathrm { t } ) \left\|_{\mathrm{L}^{2}}^{2}|\leqslant 2 \mathrm{D}|\left|\widetilde{v}_{\xi \xi}(\mathrm{t})\left\|_{\mathrm{L}^{2}}^{2}+2 \mathrm{k}_{2}|| \widetilde{v}_{\xi}(\mathrm{t})\right\|_{\mathrm{L}^{2}}^{2}+\right| \mathrm{I}_{1}(\mathrm{t})|+| \mathrm{I}_{2}(\mathrm{t})|+| \mathrm{I}_{3}(\mathrm{t}) \mid .\right.\right.
$$

Integrating it with respect to $t$ over $[0, t]$, we get

$$
\int_{0}^{t}\left|\frac{d}{d s}\left\|\widetilde{v}_{\xi}(s)\right\|_{L^{2}}^{2}\right| d s \leqslant C_{7}\left(M_{\infty}+1\right)\left(\left\|\widetilde{v}_{0}(0)\right\|_{\mathrm{H}^{1}}^{2}+\int_{-\tau}^{0}\left\|\widetilde{v}_{0}(s)\right\|_{\mathrm{H}^{1}}^{2} \mathrm{ds}\right) \leqslant \mathrm{C}_{7}\left(\mathrm{M}_{\infty}+1\right) \mathrm{M}_{0}^{2}
$$

for some constant $C_{7}>0$, provided $M_{\infty} \leqslant \delta_{1}$. This proves (4.11).

We now prove the boundedness for $\|v(t)\|_{C}=\left\|w^{-\frac{1}{2}} \widetilde{v}(t)\right\|_{C}$ uniformly in $t \in[0, \infty)$. Since $v \in X(0, \infty)$, so $v \in \mathrm{C}_{\text {unif }}(0, \infty)$, namely, $\lim _{\xi \rightarrow+\infty} v(t, \xi)=v(t, \infty)=: z(t)$ exists uniformly for $t \in[-\tau, \infty)$, and $\lim _{\xi \rightarrow+\infty} v_{\xi}(t, \xi)=\lim _{\xi \rightarrow+\infty} v_{\xi \xi}(t, \xi)=0$ are uniformly for $t \in[-\tau, \infty)$. Let us take the limits to (2.2) as $\xi \rightarrow+\infty$, then

$$
\left\{\begin{array}{l}
z^{\prime}(\mathrm{t})+\mathrm{d} z(\mathrm{t})-\mathrm{b}^{\prime}\left(\mathrm{u}_{+}\right) z(\mathrm{t}-\tau)=\mathrm{Q}(z(\mathrm{t}-\tau)) \\
z(\mathrm{~s})=z_{0}(\mathrm{~s}), \quad s \in[-\tau, 0] .
\end{array}\right.
$$

Similarly to [1] and [6], we have the following results.

Lemma 4.5. When $\mathrm{d} \geqslant \mathrm{b}^{\prime}\left(\mathrm{u}_{+}\right)$, with any time-delay $\tau>0$, or $\mathrm{d}<\mathrm{b}^{\prime}\left(\mathrm{u}_{+}\right)$but with a small time-delay $0<\tau<\bar{\tau}$, where $\bar{\tau}$ is a constant, then,

$$
|u(t, \infty)|=|z(t)| \leqslant \mathrm{CM}_{0} e^{-\mu t}, \quad t>0
$$

for some $0<\mu=\mu\left(\mathrm{p}, \mathrm{d}, \tau, \mathrm{b}^{\prime}\left(\mathrm{u}_{+}\right)\right)<\mathrm{d}$, provided with $\left|z_{0}\right| \ll 1$.

Lemma 4.6. It holds that

$$
\|\mathrm{u}(\mathrm{t})\|_{\mathrm{C}} \leqslant \mathrm{C}_{8} \sqrt{\mathrm{M}_{\infty}+1} \mathrm{M}_{0}, \quad \mathrm{t} \in[0, \infty)
$$

provided $\mathrm{M}_{\infty} \leqslant \delta_{1}$.

Combining Lemma 4.3, Lemma 4.4, and Lemma 4.6, we can immediately prove Theorem 2.2.

\section{Proof of asymptotic stability}

From (2.3) and (4.11), when $M_{0} \leqslant \delta_{0}$, we get

$$
\begin{aligned}
& \|v(t)\|_{C}+\|(\sqrt{w} v)(t)\|_{\mathrm{H}^{1}}^{2}+\int_{0}^{t}\|(\sqrt{\phi w} v)(s)\|_{\mathrm{L}^{2}}^{2} \mathrm{~d} s+\int_{0}^{\mathrm{t}}\left\|(\sqrt{w} v)_{\xi}(s)\right\|_{\mathrm{H}^{1}}^{2} \mathrm{~d} s+\int_{0}^{\mathrm{t}}\left|\frac{\mathrm{d}}{\mathrm{ds}}\|(\sqrt{w} v) \xi(s)\|_{\mathrm{L}^{2}}^{2}\right| \mathrm{d} s \\
& \quad \leqslant \mathrm{C}\left(\mathrm{M}_{\infty}+1\right) \mathrm{M}_{0}^{2}, \quad \mathrm{t} \in[0, \infty) .
\end{aligned}
$$

Set $f(t):=\left\|\widetilde{v}_{\xi}\right\|_{L^{2}}^{2}=\|(\sqrt{w} v) \xi(s)\|_{L^{2}}^{2}$. From (5.1), we get that

$$
0 \leqslant f(t) \leqslant C M_{0}^{2}, \quad \int_{0}^{\infty} f(t) d t \leqslant C M_{0}^{2}, \quad \int_{0}^{\infty}\left|f^{\prime}(t)\right| d t \leqslant C M_{0}^{2} .
$$


This implies

$$
\lim _{t \rightarrow \infty} f(t)=0 \text {, i.e., } \lim _{t \rightarrow \infty}\left\|\widetilde{v}_{\xi}\right\|_{L^{2}}^{2}=0 .
$$

By using Sobolev inequality $\mathrm{H}^{1}(\mathbb{R}) \hookrightarrow \mathrm{C}(\mathbb{R})$

$$
\|\widetilde{v}(t)\|_{C} \leqslant \sqrt{2}\|\widetilde{v}(t)\|^{\frac{1}{2}}\left\|\widetilde{v}_{\xi}(t)\right\|^{\frac{1}{2}},
$$

and the boundedness of $\|\widetilde{v}(t)\|=\|(\sqrt{w} v)(t)\| \leqslant C M_{0}$ and the convergence of (5.2), we then prove

$$
\lim _{t \rightarrow \infty} \sup _{\xi \in \mathbb{R}}|\sqrt{w(\xi)} v(t, \xi)|=\lim _{t \rightarrow \infty} \mid \widetilde{v}(t) \|_{C}=0 .
$$

Now, we are going to prove the convergence of $\lim _{t \rightarrow \infty} \sup _{\xi \in \mathbb{R}}|v(t, \xi)|=0$. To prove such a stability relation, let us start from the far field $\xi \gg 1$. By the same fashion as shown in lemma (4.6), the solution $z(t)=v(t, \infty)$ to the delayed ODE (4.16) decays exponentially

$$
|v(t, \infty)|=|z(t)| \leqslant \mathrm{CM}_{0} e^{-\mu t}, \quad t \in[0, \infty) .
$$

When $d \geqslant b^{\prime}\left(u_{+}\right)$with arbitrary time-delay $\tau>0$, or $d<b^{\prime}\left(u_{+}\right)$but with a small time-delay $0<$ $\tau<\bar{\tau}$. According to (2.1), we can write the solution in the integral form represented by the heat kernel $G(t-s, \xi-\eta):$

$$
\begin{aligned}
v(t, \xi)= & e^{-d t} \int_{R} G(\eta, t) v(0, \xi-\eta) d \eta+\int_{0}^{t} e^{-d(t-s)} \int_{R} G(\eta, t-s) \cdot \int_{\mathbb{R}} h(y) \\
& \cdot P\left(v\left(s-\tau, \xi-\eta-y-c_{*} \tau\right)\right) d y d \eta d s .
\end{aligned}
$$

Multiplying (5.5) by $e^{\mu t}$ and noting $|\mathrm{P}(v)| \leqslant C|v|, i=1,2$, then we have

$$
\begin{aligned}
\left|e^{\mu t} v(t, \xi)\right| \leqslant & e^{-(d-\mu) t} \int_{R} G(\eta, t)\left|v_{0}(0, \xi-\eta)\right| d \eta+e^{\mu t} \int_{0}^{t} e^{-d(t-s)} \int_{R} G(\eta, t-s) \\
& \cdot \int_{\mathbb{R}} h(y)\left|P\left(v\left(s-\tau, \xi-\eta-y-c_{*} \tau\right)\right)\right| d y d \eta d s \\
\leqslant & e^{-(d-\mu) t} \int_{R} G(\eta, t)\left|v_{0}(0, \xi-\eta)\right| d \eta+C e^{\mu t} \int_{0}^{t} e^{-d(t-s)} \int_{R} G(\eta, t-s) \\
& \cdot \int_{\mathbb{R}} h(y)\left|v\left(s-\tau, \xi-\eta-y-c_{*} \tau\right)\right| d y d \eta d s .
\end{aligned}
$$

Since $v \in X(0, \infty)$ is the global solution of (2.2), namely $v \in C_{\text {unif }}(0, \infty)$, then $v(t, \xi) \rightarrow v(t, \infty)=z(t)$ as $\xi \rightarrow \infty$ uniformly in $t \in[0, \infty)$. By applying the property of the heat kernel and the exponential decay (5.4), then from (5.6) we get

$$
\begin{aligned}
\lim _{\xi \rightarrow \infty}\left|e^{\mu t} v(t, \xi)\right| \leqslant & e^{-(d-\mu) t} \int_{R} G(\eta, t) \lim _{\xi \rightarrow \infty}\left|v_{0}(0, \xi-\eta)\right| d \eta+C e^{\mu t} \int_{0}^{t} e^{-d(t-s)} \\
& \cdot \int_{R} G(\eta, t-s) \int_{\mathbb{R}} h(y) \lim _{\xi \rightarrow \infty}\left|v\left(s-\tau, \xi-\eta-y-c_{*} \tau\right)\right| d y d \eta d s \\
\leqslant & \left|v_{0, \infty}(0)\right| e^{-(d-\mu) t}+C e^{\mu t} \int_{0}^{t}|z(s-\tau)| d s \\
\leqslant & \left|v_{0, \infty}(0)\right| e^{-(d-\mu) t}+C e^{\mu t} \int_{0}^{t} e^{-d(t-s)} e^{-\mu(s-\tau)} d s \\
= & \left|v_{0, \infty}(0)\right| e^{-(d-\mu) t}+\frac{C e^{\mu \tau}}{d-\mu}\left(1-e^{-(d-\mu) t}\right) \\
\leqslant & C, \text { uniformly in all } t>0 .
\end{aligned}
$$


This quickly implies that, there exists a number $x_{1} \gg 1$ (independent of $t$ ), such that when $\xi \geqslant x_{1}$, then

$$
\sup _{\xi \in\left[x_{1}, \infty\right)}|v(t, \xi)| \leqslant C e^{-\mu t}, \quad t>0
$$

Note that, $\sqrt{w(\xi)}=e^{-\lambda_{*} \xi} \geqslant e^{-\lambda_{*} x_{1}}$ for $\xi \in\left(-\infty, x_{1}\right]$, then (5.3) implies

$$
\lim _{t \rightarrow \infty} \sup _{\xi \in\left[x_{1}, \infty\right)}|v(t, \xi)| \leqslant \lim _{t \rightarrow \infty} \sup _{\xi \in\left[x_{1}, \infty\right)}\left|\frac{\sqrt{w(\xi)}}{e^{-\lambda_{*} x_{1}}} v(t, \xi)\right| \leqslant e^{\lambda_{*} x_{1}} \lim _{t \rightarrow \infty} \sup _{\xi \in \mathbb{R}}|\sqrt{w(\xi)} v(t, \xi)|=0 .
$$

This with (5.7) together proves

$$
\lim _{t \rightarrow \infty} \sup _{\xi \in \mathbb{R}}|v(t, \xi)|=0
$$

The proof is complete.

\section{References}

[1] I.-L. Chern, M. Mei, X. Yang, Q. Zhang, Stability of non-monotone critical traveling waves for reaction-diffusion equations with time-delay, J. Differential Equations, 259 (2015), 1503-1541. 1, 1, 4, 4

[2] A. Gomez, S. Trfimchuk, Monotone traveling wavefronts of the KPP-Fisher delayed equation, J. Differential Equations, 250 (2011), 1767-1787. 1

[3] S. A. Gourley, J. W.-H. So, J. H. Wu, Non-locality of reactionCdiffusion equations induced by delay: biological modeling and nonlinear dynamics, J. Math. Sci., 124 (2004), 5119-5153. 1

[4] S. A. Gourley, J. Wu, Delayed nonlocal diffusive system in biological invasion and disease spread, Fields Inst. Commun., Am. Math. Soc., 48 (2006), 137-200. 1

[5] R. Huang, M. Mei, K. Zhang, Q. Zhang, Stability of oscillating wavefronts for time-delayed nonlocal dispersal equations, Disc. Continu. Dynam. Sys.-Ser. A., 36 (2016), 1331-1353. 4

[6] C.-K. Lin, C.-T. Lin, Y. Lin, M. Mei, Exponential stability of nonmonotone traveling waves for Nicholson's Blowfilws equation, SIAM. J. Math. Anal., 46 (2014), 1053-1084. 1, 4, 4

[7] C.-K. Lin, M. Mei, On traveling wavefronts of the Nicholson's blow ies equation with diffusion, Proc. Roy. Soc. Edinburgh, 140 (2010), 135-152. 1

[8] A. Matsumura, M. Mei, Nonlinear stability of viscous shock profile for a non-convex system of viscoelasticity, Osaka J. Math., 34 (1997), 589-603. 4

[9] M. Mei, Stability of traveling wavefronts for time-delay reaction-diffusion equations, Discrete Contin. Dyn. Syst. Supplement, 2009 (2009), 10 pages.

[10] M. Mei, C.-K. Lin, C.-T. Lin, J. W.-H. So, Traveling wavefronts for time-delayed reaction-diffusion equation: (I) Local nonlinearity, J. Differential Equations, 247 (2009), 495-510. 1, 1, 1, 4

[11] M. Mei, C.-K. Lin, C.-T. Lin, J. W.-H. So, Traveling wavefronts for time-delayed reaction-diffusion equation: (II) nonlocal nonlinearity, J. Differential Equations, 247 (2009), 511-529. 1

[12] M. Mei, C. Ou, X.-Q. Zhao, Global stability of monostable traveling waves for nonlocal time-delayed reaction-diffusion equations, SIAM J. Math. Anal., 42 (2010), 2762-2790. 1

[13] M. Mei, C. Ou, X.-Q. Zhao, Global stability of monostable traveling waves for nonlocal time-delayed reaction-diffusion equations, SIAM J. Math. Anal., 44 (2012), 538-540. 1

[14] M. Mei, Y. Wang, Remark on stability of traveling waves for nonlocal Fisher-KPP equations, Int. J. Numer. Anal. Model. Ser. B, 2 (2011), 379-401. 1

[15] H. J. K. Moet, A note on asymptotic behavior of solutions of the KPP equation, SIAM J. Math. Anal., 10 (1979), 728-732. 1,4

[16] J. W.-H. So, X. Zou, Traveling waves for the diffusive Nicholson's blowflies equation, Appl. Math. Comput., 122 (2001), 385-392. 1

[17] E. Trofimchuk, M. Pinto, S. Trofimchuk, Pushed traveling fronts in monostable equations with monotone delayed reaction, Discrete Contin. Dyn. Syst., 33 (2013), 2169-2187. 1

[18] Z. Xu, D. Xiao, Spreading speeds and uniqueness of traveling waves for a reaction diffusion equation with spatio-temporal delays, J. Differential Equations, 260 (2016), 268-303. 1 